yf car su-f/yf. $\mathrm{Dp}\left(s \mathrm{~V}^{\mathrm{V}} y^{+}\right)$with or without the autosomal inversions, SMl and Ubx ${ }^{180}$, were crossed to $y$ f car $s u=f / Y$ males and the $F_{1}$ scored for recombination.

It can be seen from Table 1 that the car-su-f region which includes euchromatin exhibits a pronounced increase in recombination in the presence of the autosomal inversions while the region which is heterochromatic is refractory to the interchromosomal effect. The interchromosomal effect on recombination which has hitherto been described as most pronounced in "heterochromatic regions' is more accurately described as pronounced in the proximal euchromatin but absent in heterochromatin.

Table 1. Racombination IN EU- AND Hetero-chromatin OF THE $X$

Autosomal inversions present

None
SMl; Ubx

Cro. of
No.es
fcored
5,005
9,278
Chrome

Eu
Per cen
cartromati
$\mathbf{8 . 5 4}$ eterochromatic $8 u-f-y^{+}$ 0.04
0.04

Crossing-over apparently occurs in the early growth stages of the oocyt $\theta^{2}$. In spite of the generally unfavourable nature of the cytological material, heterochromatin is demonstrable in the oocyte nucleus throughout these early growth stages. Moreover, the nucloolus organizer is in $\vec{X}$ heterochromatin, and when the nucleolus is first visible (stage 3) and in succeeding stages it is seen to be associated with heterochromatin ${ }^{3}$. It has recently been demonstrated that condensed, pycnotic chromatin is relatively inactive in the synthesis of RNA compared with the more diffuse chromatin ${ }^{4}$. The results recorded here make it possible to correlate another aspect of chromosome behaviour with a particular cytological state and indicate a fundamental difference in the exchange behaviour of eu- and hetero-chromatin owing, apparently, to the condensed state of heterochromatin at the time of exchange. It follows that the more diffuse chromatin condition is a prerequisite for tho regular and frequent exchanges that occur during meiosis.

This work was sponsored by the U.S. Atomic Fnergy Commission under contract with the Union Carbide Corporation.

Biology Division,

Oak Ridge National Laboratory, Oak Ridge, Tennossee.

${ }^{1}$ Cooper, K. W., Chromosoma, 10, 535 (1959).

' Plough, H. H., J. Exp. Zool, 24, 148 (1917).

King, R. C., Rubinson, A. C., and Smith, R. F., Growth, 20, 121 (1956).

- Littau, V. C., Allfrey, V. G., Frenster, J. H., and Mirsky, A. E., Proc. U.S. Nat, Acad. Sci., b2, 93 (1964).

\section{Pseudocholinesterase Variants in Japan}

Since Kalow ${ }^{1}$ in 1956 demonstrated a genotically controlled atypical (dibucaine resistant) variant of human pseudocholinesterase (acylcholine acylhydrolaso Enzyme Commission: 3.1 .1 .8$]$ ) much information has been gained on the polymorphism of this enzyme, an esterase of the serum of which the function is not known. Besides the most common gene $\left(C h_{1} \bar{V}\right)$ responsible for the synthesis of the usual pseudocholinesterase at least three other genes have been described on the same autosomal locus, namely, the alleles $C h_{1} D, C h_{1} F$ and $C h_{1} s$, each producing variant enzymes different from the normal pseudocholinesterase. There is no difference in health between various genotypes of the usual gene $C h_{1} U$ and those of the alleles $C h_{1} D, C h_{1} F^{\prime}$ and $C h_{1} S$, unless either homozygotes or heterozygotes of each of the latter three genes are subjected to the application of the muscle relaxant suxamethonium (succinyldicholin) when an abnormally prolonged apnoea is observed. Heterozygotes with both a variant gene and the usual gene do not show this sensitivity.

Various methods have been developed to identify the different types of the enzyme in the homozygotes as well as in the heterozygotes. Among the inhibition-tests the most common are those using dibucaine ${ }^{2}$ or $\mathrm{NaF}^{\mathrm{s}}$ as inhibitors.

The incidence of the heterozygotes for the atypical allele $C h_{1} D$ in Europe and Canada seems to be almost constant among various populations so far investigated: about 3-4 per cent4-7. Recent investigations on 433 Israelites suggests a higher frequency of the gene $C h_{1} D$ (6.3 per cent) among them ${ }^{8}$. Australian Aborigines and Malaysians are said to show no significant difference from the European populations ${ }^{9,10}$. Consequently the atypical type $\left(C h_{1} D\right)$ of pseudocholinesterase is believed to have a remarkable uniformity of incidence in widely different populations, suggesting that there are (or have been) differences between the selective values of pseudocholinesterase-variants of the various populations ${ }^{10-12}$. Investigations on the incidence of the gene $C h_{1}{ }^{F}$ (fluoride resistant) suggests it has about the same frequency as that of the gene $C h_{1}{ }^{D}$ (refs. 7, 12). Homozygotes for the "silent gene' $O h_{1} S$ are supposed to be found one in 100,000 (ref. 11). We have recently investigatod 100 serum samples from Japan, randomly collected in Tokyo from healthy blood donors. The wholo material was investligated at first by two screening tests, the diffusion-test ${ }^{18,14}$ and the inhibition-test with $R O 2-0683$ as inhibitor according to $K_{\text {alow }}{ }^{15}$. The dibucaine and fluoride numbers were measured afterwards on each of the 100 sera. Neither heterozygotes nor homozygotes for the gene $C h_{1} D$ were found by the four methods described, while two sera were found to be heterozygous for the gene $C h_{1}{ }^{F}$.

The esterase level in our material was found to be lower than that of other populations. We do not believe that the depressed enzyme activity can be explained by a five days' transport from Japan to our laboratory at uncontrolled temperature, but further investigations will be necessary. Both the dibucaine and the fluoride numbers showed normal values.

The foregoing result was confirmed by the screening tests ${ }^{13-15}$ on a further 150 Japanoso sera, in which no $C h_{1}{ }^{n}$ phenotype was found. The frequency of the heterozygotes for $C h_{1}{ }^{F}$, on the other hand, seemed to be about the same as found in the earlier investigation. This may suggest that the incidence of the gene $C h_{1} D$ is not as evenly distributed as was assumed from earlier investigations.

This work was supported by the Alexander von Hum. boldt-Stiftung, the Deutsche Forschungsgemeinschaft and the Bundesministerium für Wissenschaftliche Forschung, Bad Godesberg.

We thank Dr. H. Sakura, Department of Legal Medicine, Medical Dental University, Tokyo, for sending us the sera.

K. Омото H. W. GOEDDE

Section of Biochemical Genetics,

Institute of $\Lambda$ nthropology,

University of Freiburg i. Br., Germany.

${ }^{\prime} \mathrm{K}$ alow, W., Lancet, ii, 570 (1956).

${ }^{2}$ Kalow, W., and Genest, K., Canad. J. Biochem. Physiot., 35, 339 (1957).

${ }^{3}$ Harris, H, and Whittaker, M., Nature, 191, 496 (1961).

4 Kalow, W., and Gurin, D. R., Ann. Hum. Genet., 23, 239 (1958).

- Kattamis, Chr., Zannos-Mariolea, L., Franco, A. P., Liddell, H., Lehmann, II., and Davies, D., Nature, 196, 599 (1962).

${ }^{6}$ Gocdde, H. W., and Altland, K., Nature, 198, 1208 (1968).

7 Goedde, II. W., Altland, K., and Bross, K., Dtsch. med. Wschr., 88, 2510 (1963). 8zeinberit, A., Meyer, M, , Fisenberg, Z., Ostfeld, E., Bar-Or, R., and Ezra,
R., Istael med. J, g2, 137 (1963).

- Horsfall, W. R., Jehmann, H., and Davieg, D., Nature, 199, 115 (1968).

10 Lehmanin, H., and Lildell, J., in Progress in Medical Genetics, edit. by Steinberg, A. G., and Bearn, A. G., 3 (Grune and Stratton, New York and Lotion, 1964).

11 Motulsky, A. G., Progress in Medical Genetics, 49 (1964).

12 Goedde, H. W., and Schoepf, E., Deutsche Med. Klinik, 59, 1849 (1964).

is Harris, $H_{i ;}$ and Robson, E. B., Lancet, ii, 218 (1963).

${ }^{14}$ Goedde, H. W., and Fuss, W., Klin. Wschr, 42 (6), 286 (1964).

${ }^{15}$ Kalow, W., and Davies, R. O., Biochem. F'harmacol, 1, 183 (1959), 